\title{
Source and Sink Theory
}

\author{
Mohsen Sharifpur \\ Department of Mechanical and Aeronautical, University of Pretoria, Pretoria 0002, South Africa \\ Email: mohsen.sharifpur@up.ac.za
}

\begin{abstract}
The lack of information from the very early Universe and offering a new theory to answer the unanswered questions has long been a topic of great interest. One of the most important questions is about the origin of the Dark Energy which causes the acceleration of the galaxies as well as the expansion of the Universe. Inflation theory tried to sort out of problems with Big Bang cosmology, but couldn't, and that's why there are different versions of inflation theory. Therefore, the lack of a new and general theory to answer the unanswered questions is obvious. If Big Bang is a part of nature, the knowledge of different patterns in nature like fractional geometries and Fibonacci, fluid dynamics, constructal law needs to be considered for the new theory. In nature, everything behaves as a pattern of a flow (which is approved by constructal law), as well as, whatever we have in nature has a pair (like male and female), or opposite (like opposite charges of electron and positron), or poles (like a magnetic field), ... In this way, by using the similarity of the cases and elaborately investigate into nature, it is possible to propose a new theory to answer the unanswered questions. The "Source and Sink Theory", simply indicates that "if there is a source, it must be at least one sink (at the same instance), with a flow pattern from source to the sink, and the process must be conserved". By applying this general theory to our Universe, it could indicate that the so-called Big Bang could be a pulse of a point source and at the same instance, it should be at least an opposite of the Big Bang as a sink. Consequently, the energy which introduced from our Universe origin (Big Bang) as a pulse of a point source with a unique cosmic microwave background radiation flows toward a sink (or sinks). A sink has gravity (curvature of space-time) which could produce a flow pattern for the energy released from source to the sink. The cosmic microwave background radiation could also support this theory as much as it supported the Hot Big Bang model. In the special case (set the gravity of the sink to zero, and no space between source and sink), application of this theory for cosmology will switch back to Big Bang cosmology, which shows more ability of this theory to produce different scenarios to fit the available data. Application of this general multi-discipline theory on our Universe can also offer an answer to some of the unanswered questions like the origin of Dark Energy, galaxies acceleration, so-called expansion of the Universe and much more...
\end{abstract}

Keywords: Big Bang, inflation, constructal law, Dark Energy, fractional geometry, cosmic microwave background radiation.

\section{Introduction}

To date, there has been evaluated some different theories of the early Universe which the important ones are the steady-state universe [1] (always expanding with constant average density), the cyclic universe [2] (oscillatory Universe interpretation of the BB), and the standard Big Bang (BB) theory [3]. Almost all the astronomical data is in favour of Big Bang cosmology, however, there is good evidence to believe that the standard model (Lambda-CDM) is not the end of the story with some unanswered questions [4]. However, one of the models which can explain the best the data from Planck satellite [5] is the inflation theory [6]. Inflation theory invented to sort out the problems with Big Bang cosmology, and it was successful in some aspects but not on some others, that's why different versions of inflation theory developed. There are more than ten different versions of inflation theory as; Old Inflation, Chaotic Inflation, New Inflation, Internal Inflation, Sugra Inflation, Extended Inflation, Power-Law Inflation, Double Inflation, Hybrid Inflation, Natural Inflation, Brane Inflation, DBI Inflation, N-Flation, SUSY F-term Inflation, SUSY D-term Inflation, SUSY P-term Inflation, Assisted Inflation, K-Flation, and Warped Brane Inflation. All versions of inflation theory are almost common about eternal to the future but finite to the past. It is hard to find one which has origin and eternal to the past (they are 
different concerning if there was a beginning of the time and if there was a singularity). However, Aguirre and Gratton extended the inflation theory which no needs a big bang [7]. There is also some evidence showing the necessity for alternative models to inflation [8].

The inflation theory, in general, has an explanation about an isotropic Universe in all directions, the distribution of the cosmic microwave background radiation, the flat Universe, and why the magnetic field is bipolar. However, the detailed particle physics mechanism responsible for inflation is unknown. Both BB and standard inflation theories supported the idea that everything started after the BB and nothing was before BB. This has a conflict with the conservation of energy; also, if the BB happened without any reason, it must happen again! When the scientists found out the BB theory does not work properly or couldn't explain the real size of available Universe, they did not doubt it; they try to fix it with other theories like Inflation.

On the other hand, Dark Energy (DE) which caused the accelerated expansion of our Universe [9] is still an unknown phenomenon. Therefore, the presently available theories (in the open literature) cannot clearly answer the following questions:

- Is the BB a part of nature? And if so, is there any pattern for it?

- Where is the possible origin of the energy of the BB?

- What was the condition of our Universe before the BB?

- What is the origin of the Dark Energy?

- Explanation on the so-called expansion of the Universe.

If we look at nature elaborately, we can find everything has a pair (like male and female), or they are negative and opposite (like electron and proton, or electron and positron), or they are bipolar (magnetic poles). Also, we accepted the existence of matter and antimatter. Therefore, if we had a Big Bang, we can think about the possibility of a pair or an opposite for it.

Fractal geometry has approved that there are patterns in nature [10-12]. On the other hand, Adrian Bejan by constructal law [13] showed that there are flow patterns in nature (like a pattern of fluid flow in fluid mechanics) for trees, tributaries, air passages, neural networks, and lightning bolts, and much more cases. Biology, physics, technology, and social organizations obey a single principle of physics. The constructal law accounts for the evolution of them and many other designs in our world.

Earth, solar system, our galaxy, and whatever we can observe in our Universe are moving like a pattern of fluid flow. The human body, the growing of the plants, tornado, departing the continents from each other in the Earth, the pattern of the Earth's magnetic field and the shielding from the geomagnetic storms, the path of the stars, stellar remnants, interstellar gas and dust around galaxies ... all of them are like a fluid flow pattern as well. Therefore, it is also the possibility to extend these flow patterns in nature from the beginning of BB to build a general theory. On the other hand, the availability of patterns in nature and the Universe like the Fibonacci pattern [14, 15] allowed us to employ a pattern in our predictions for the Universe.

\section{Flow Patterns in Nature}

As indicated in the introduction, if we elaborately investigate nature, we can find different patterns which they look like a pattern of fluid flow. There are a lot of patterns in nature which they investigated by researchers in constructal law [13, 16], fractal geometry [10-12], Fibonacci [14, 15] ... to date. Here, I would also like to add a new point of view which is not reported in the open literature.

Plants and trees are the most common important part of nature. If we look at Fig. 1, which is a section of a tree's wood along the stem, we can recognize that it looks like a fluid flow pattern. Even the wood knots (the place of the branches at the trees' wood) look like a pattern of fluid flow. Fig. 2-a shows the section of two different trees' wood along with the steam at the place of a branch (wood knot) and Fig.2-b is the simulation of fluid (water) flow in a vertical cylinder passing over a horizontal cylinder. From Figs.1 and 2, it is possible to conclude that the pattern of growing the trees' wood looks like a fluid flow pattern as well.

Also, this can open a new window for research to investigate nature. In this way, like the fluid flow which is possible to predict the flow behaviour by using Reynolds Number (Re) [17], it is possible to investigate for a compatible Re for the flow pattern in the trees' wood, or plants. Fig. 2 tries to show the patterns, and if the condition of the simulation gets more matched with the condition of the wood 
(size of the tree, size of the branch, angle of the branch...), the result of the simulation could be more matched (which is another on-going research and out of the subject of this manuscript).

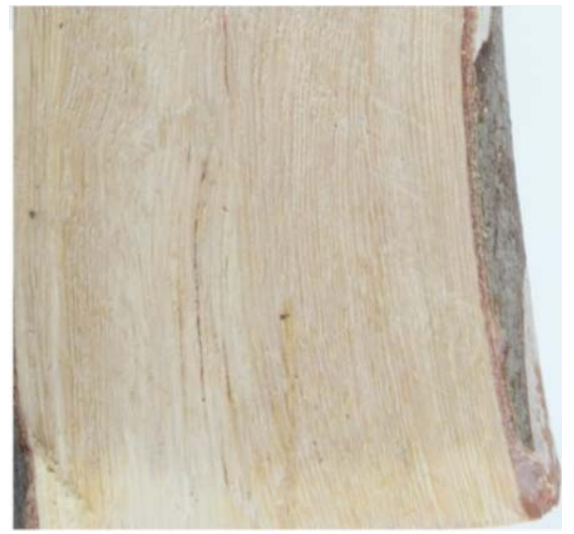

Figure 1. Flow pattern in a wood stem.
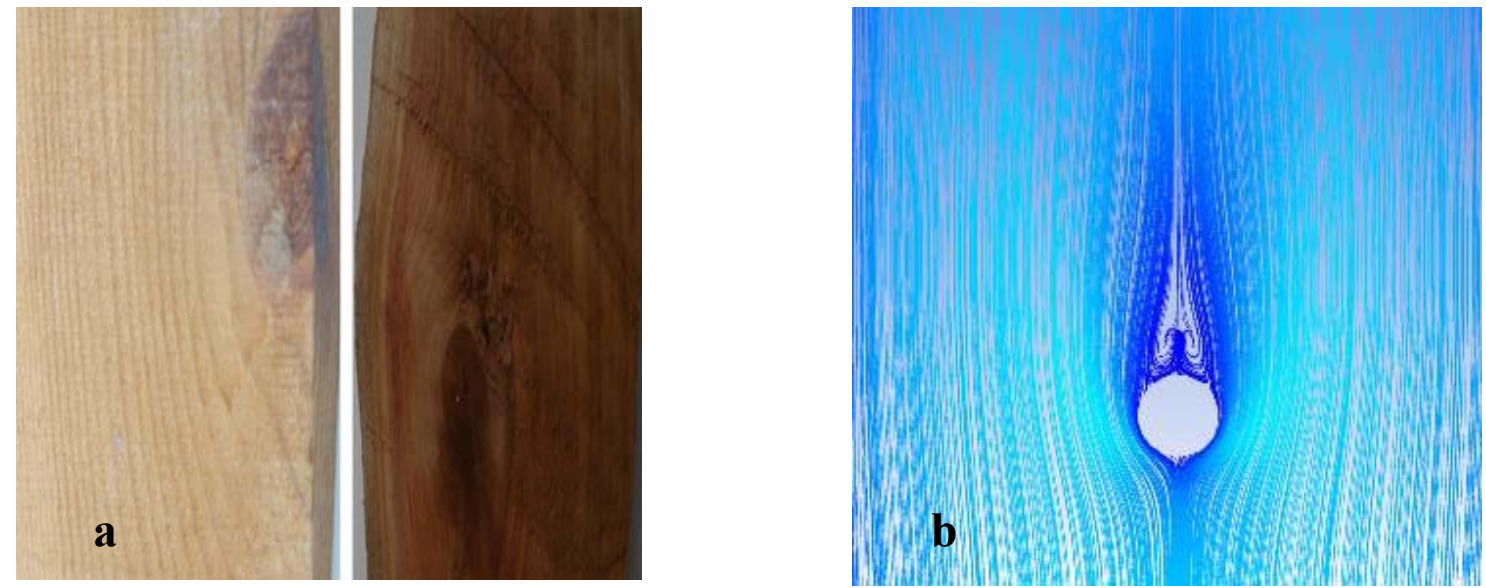

Figure 2. a) Flow pattern in two wood sections from two different trees along the stem at the place of a branch (wood knot) b) simulation of fluid flow (water) in a vertical cylinder passing over a horizontal cylinder.

\section{Source and Sink Theory}

The reader should be informed that this theory is far from being complete and that some important aspects are to be discussed and to be completed with the efforts of the scientists of different fields. However, the answer to some of the unknown queries achieved up to the present has been motivating me to share the idea publicly.

As indicated in the introduction, everything in nature has a pair, or negative side, or opposite pole or an anti. Also, if there is a source, there will be a sink which they connected by a flow pattern. For example, if there is a hot object as a source of heat transfer, the heat will transfer (naturally) to any cold object around it (as a sink) by natural convection [18]. On the other hand, constructal law [13] approved that there are flow patterns in nature. Different patterns in nature like fractal geometry [10-12] and Fibonacci $[14,15]$ provide us with a hint to think on a pattern from the beginning of the Universe.

The "source and sink (SS) theory" indicates, "If there is a source, it must be at least one sink (at the same instance) with a flow pattern from the source to the sink, and the process must be conserved". In this way, if we apply this theory for the early universe, the origin of our Universe (BB) could be as a point source which produces a pulse of energy. Therefore, it must be at least one sink which the pulse goes toward the sink like a fluid flow pattern. The Black Holes (BHs) could be considered as sinks, but cosmologists believe that the BHs came to the story of cosmology after BB, and they were not available 
at the instant of BB to produce the flow direction. On the other hand, BHs (in the visible Universe) are a part of the moving pulse of our Universe point source, and they are a part of the galaxies acceleration to a sink.

Fig. 3 shows the cross-section of a very simple sketch of a point source and a surrounded sink for a homogenous Universe which the sink has gravity force (this sketch tries to offer an understanding of the idea, and in reality, the size of the point source (BB) must be very smaller which cannot be seen in the sketch at all). The sink in Fig. 3 looks like the shell of an empty sphere which the shell has gravity (space-time curvature). Therefore, the gravity of the sink (curvature of space-time) causes a flow pattern from the point source to the sink. The surrounded sink will absorb whatever emitted from the point source after each pulse by its gravity (curvature of space-time). Fig. 3 is the simplest possible idea for the sink of a homogeneous Universe like ours (if we accept that our Universe is almost homogeneous in all directions [19]). Also, it is possible to explore different scenarios for the shape of the sink(s). For example, several point sinks in a curved position could produce a homogeneous Universe as well. However, the sink required curvature to make at least a point of gravity concentration/singularity. This theory even by considering the simplest case of Fig. 3 has the potential to answer some of the previously unanswered questions as follows but bring new questions as well. After a pulse emitted from the point source (so-called BB), it has an initial velocity while near the source the equivalent resultant gravity force (vector) of the sink (so-called Dark Energy) is not considerable. When the pulse gets a bit far from the source, the resultant gravity (force) vector (curvature of space-time) of the sink can effect on different parts of the pulse to accelerate the galaxies to the sink. The pulse goes toward the sink. Therefore, the so-called expansion of the universe is obvious in this theory. We do not have a lot of information about our Universe sink except the resultant gravity force (vector) of the sink as Dark Energy. Like Black Holes, it can consider an event horizon for the sinks as well.

The Hot Big Bang (HBB) model and standard inflation theory indicated there was nothing before the BB (without any scientific reason), and that's why they are not conserved for a time interval before and after BB (in this way they had to indicate that everything began after the BB). Also, they cannot explain the Dark Energy, Dark Mater, acceleration of the galaxies and the so-called expansion of the Universe properly. On the other hand, there is evidence which inflation does not explain the HBB model's fine-tuning problems as well [20].

By SS theory, the following questions will be explained and answered even by considering the simple sketch of Fig. 3 for our Universe sink. However, it is possible to offer more complicated cases with almost the same result. This shows a curved sink or number of point sinks in a curved arrangement need to be investigated and compared for our Universe sink elaborately.

\section{1 "Source and Sink" and "Cosmic Microwave Background Radiation"}

SS theory suggests that the BB was a pulse of energy from a point source, and the pulse flows to a sink (or sinks). Therefore, in SS theory, each pulse has a unique cosmic microwave background radiation (CMBR). This means as much as the CMBR supports a Hot Big Bang (HBB) [21], it must support SS theory. After a fraction of the time from a pulse of our Universe source, the SS theory and HBB model have not any conflict on the concept of cosmic microwave background radiation. However, the main conflict between HBB and inflation with SS is before the pulse (before the BB) and what happens in the future (SS theory predicts everything in the Universe goes toward a sink (or sinks)). Whatever prescribes by BB theory after the BB can be one of the scenarios of SS theory. The SS theory can offer an answer for the unknown queries of the Universe, like Dark Energy (DE) and so-called expansion of the Universe. On the other hand, CMBR is not exactly uniform over the Universe [22], which HBB theory cannot answer it properly. As one of the scenarios of SS theory, a kind of space can consider between the source and the sink before the instant of BB which brings the opportunity to explain this anisotropy of CMBR (for example, with SS theory it is possible to think about the existence of a kind of Dark Matter (partially) between source and sink from the beginning).

\subsection{Possible Position of the Big Bang (Place of Our Universe Point Source)}

Place of the origin (point source) of our Universe (so-called BB) can be the centre of the sphere (or the centre of the curvature) of the $\operatorname{sink}(\mathrm{s})$. Therefore, there will be a concentration/singularity of gravity, 
which may cause the position of the source $(\mathrm{BB})$. The question may come, how the energy transfers from the sink to the source to provide the pulse(s). This is the question which needs to be assisted by the assistance of scientists of the field. However, after the sink absorbs the exact amount of the energy (from the inside, or maybe outside) and gets the critical point or unstable, the concentration of the gravity and/or instability of the sink may produce a pulse of energy from the point source. Also, after the critical point, the energy may transfer from another dimension (our Universe with higher dimensions has investigated by a lot of researchers as well as by string theory [23-25]) to the point source to produce the pulse. After the pulse of energy released, the Hot Big Bang model and SS theory do not have any conflict up to this age of Universe. However, HBB model cannot predict the future of the Universe, but the SS theory. Therefore, the so-called Big Bang should happen periodically as pulses, but we cannot sense them while we are a part of a certain pulse with our unique cosmic microwave background radiation. On the other hand, we do not have any information from the outer surface of the sink. It may be the sink absorbs matter from other Universes at the outer side to get the critical point or unstable. If we assume a uniform sink around our Universe (like Fig. 3), therefore, the flow of the pulse from the point source to the surrounded sink should be almost homogenous.

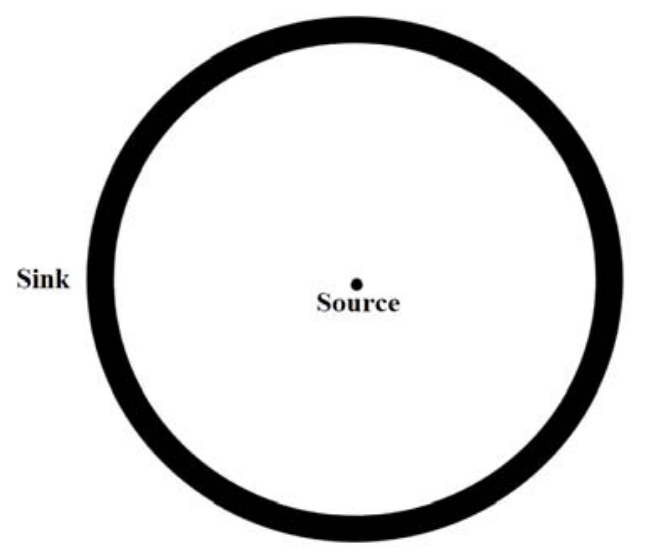

Figure 3. A very simple schematic of a point source and a surrounded sink for a homogenous Universe as one of the possible scenarios.

\subsection{Galaxies Acceleration}

When the galaxies get to an exact distance from the point source, the equivalent gravity vector toward the nearest point of the sink will be a bit considerable to accelerate the galaxies. That's why the galaxies which are much far, their speed is more. For example, in Fig. 4, a galaxy at the position A will accelerate toward point $\mathrm{B}$ at that exact instant (this does not have any conflicts with the general relativity). However, point $\mathrm{B}$ can be changed to another point at the sink if the sink is rotating. Also, there is the possibility of the rotation of the point source at the time of the injection of the pulses. Consequently, the path can be helical, like the blue curve in Fig. 4 and not strength. In this way, when a galaxy gets the point $\mathrm{C}$, the acceleration will be toward position $\mathrm{D}$, and finally will be absorbed at E. If we get back to nature, we can find another possibility for the pattern of the flow (path) from the point source to sink like a Fibonacci spiral as Fig. 5 while our nature and universe obey exact patterns $[5,6]$. In Fig. 5 just four paths of the flow pattern showed with the path (flow) pattern of the Fibonacci spiral which this case also describes a homogenous Universe, but point source or surrounded sink, or both are rotating. 


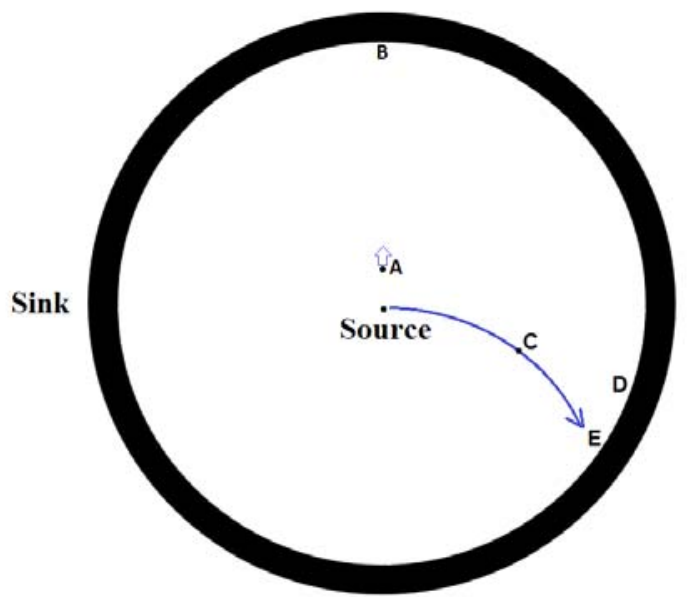

Figure 4. The galaxies close enough to the point of the sink to accelerate.

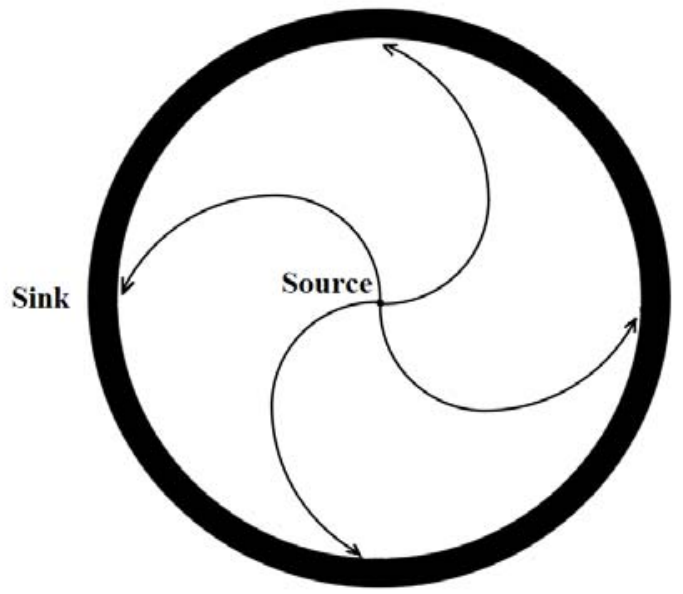

Figure 5. A point source and spherical sink with Fibonacci spiral path (pattern) flow.

\subsection{Why the Galaxies Getting Far from Each Other (So-Called Expansion of Universe)}

The point source emits a pulse to the curved sink (or point sinks in a curved arrangement), and in the case of a uniform sink (which produces uniform gravity), while the galaxies move to the sink from all directions, the so-called expansion of the Universe will happen automatically. The gravity of the sink (case of Fig.3) is from all around the sink to the galaxies. Therefore, the galaxies look like floating with partial stabilities. When the galaxies get a bit far from the point source, the resultant gravitational force toward an exact place of the sink will be more, and they accelerate toward that place (at that instant) which resulted in the so-called expansion of the universe.

\subsection{The Origin of the Dark Energy}

With SS theory, the origin of the Dark Energy will be simply the resultant gravity vector (curvature of space-time) of the curved sink (or several point sinks in a curved arrangement). Therefore, a curved sink has gravity, for example, in Fig. 4 the resultant vector of the gravity (from every point of the sink) for point $\mathrm{A}$ is a vector toward the point B (exactly at that instant). Consequently, Dark Energy is the resultant vector of the gravity force affecting that position. If a galaxy gets near to the sink, the resultant gravity vector will be more, and then more acceleration which causes more redshift [21].

\subsection{A Conserve Universe}

The $\mathrm{BB}$ and most of the versions of inflation theory are not conserved, and therefore they have to indicate that time started from the instant of $\mathrm{BB}$ and nothing was before the BB. SS theory is conserved while the energy from a sink can go to a source and from a source to a sink.

\subsection{The Bentley Paradox}

While there is a resultant vector force caused by the sink everywhere (but a bit far from the point source) and there is a flow pattern from the source to the sink. Therefore, a uniform sink will answer the Bentley paradox better.

\section{A New Question}

With SS theory, an important new question comes as: was there any matter between the source and the sink independent to the pulses (so-called BB)? It is the possibility to explore different scenarios to answer this question. Some of them can support (or partially support) the inflation theory and some 
others not. However, if there were a point source and a curved sink (or several point sinks in a curved position arrangement) from the beginning, there is the possibility to think about a kind of space between the point source and the sink. Therefore, it is possible to state the different scenarios in this regard (including a scenario to support the inflation theory).

\section{Source and Sink Theory, Big Bang Theory and Inflation Theory}

The source and sink theory could be a superior theory for Big Band and Inflation theories while it can switch to BB theory in a special case. If the gravity of sink sets to zero and consider no any kinds of space between the sink and the source, the SS theory will switch to BB theory, and needs inflation theory for the available size of our Universe. However, SS theory has the potential to offer a lot of different scenarios concerning the size and shape of source(s), $\operatorname{sink}(\mathrm{s})$ and the space between them to match the available cosmological data better. On the other hand, SS theory is a multidiscipline general theory which one of the applications could be in cosmology.

\section{Source and Sink Theory and Black Holes}

Black holes at the centre of galaxies are a kind of sink in our Universe. Also, they can be a short-cut to a sink or the surrounded sink, but with the available cosmological data, they are a part of a pulse of the point source and moving toward the carved sink (or point sinks in a carved arrangement) as a part of the flow pattern.

\section{Simple Mathematics for a Simple Source}

We do not have enough information about the curved sink (or point sinks in a carved arrangement) of our Universe, but at least most of the theories about early Universe agree with a point source like socalled Big Bang.

Assuming $t=0$ sets for one pulse of a point source. Equation (1) is the continuity equation in the spherical coordinate [27] which does not have any limitation to apply for any state of the matter.

$$
\frac{\partial \rho}{\partial t}+\frac{1}{r^{2}} \frac{\partial\left(\rho r^{2} u_{r}\right)}{\partial r}+\frac{1}{r \sin \theta} \frac{\partial\left(\rho u_{\theta} \sin \theta\right)}{\partial \theta}+\frac{1}{r \sin \theta} \frac{\partial\left(\rho u_{\varphi}\right)}{\partial \varphi}=0
$$

where $\rho(r, \theta, \varphi, t)$ is the density, and different $u$ denotes the velocities in different directions $(r, \theta, \varphi)$ which the velocities are also time-dependent. Therefore, we can employ it for any state of the Universe, except $t=0$. At exactly $t=0$, we have the case of singularity for our Universe (while $r$ goes to zero, and density goes to infinity, except if we assume an initial radius for our Universe at the instant of BB), but it is the possibility to write the continuity equation for a very small fraction of the time after the pulse (BB) for the compressible three dimensions.

For simplicity, it can assume that the velocity is just in the radial direction from the source as

$$
\frac{\partial \rho}{\partial t}+\frac{1}{r^{2}} \frac{\partial\left(\rho r^{2} u_{r}\right)}{\partial r}=0, u_{\theta}=0, u_{\varphi}=0
$$

To solve equation (2) we need some more information from the very early Universe, however, to show a simple schematic of a point source to the readers, Fig. 6 is produced for a $2 \mathrm{D}$ incompressible, and therefore, Eq. (2) reduces to Eq. (3) as

$$
u_{r}=\frac{m}{2 \pi r}, u_{\theta}=0
$$

where $m$ is the volume flow rate per unit depth for the pulse, and the point source is irrotational while there is just radial velocity as non-zero.

The stream function $\psi$ and potential function $\phi$ can be written in the complex system for this case as

$$
\phi+i \psi=r e^{i \theta}=\frac{m}{2 \pi} \ln (x+i y)
$$

which the definition of them are as follows [17] (Also, can be found in most of the Fluid Mechanics 
textbooks)

$$
u_{x} \equiv \frac{\partial \psi}{\partial y}, u_{y} \equiv-\frac{\partial \psi}{\partial x}, u_{x} \equiv \frac{\partial \phi}{\partial x}, u_{y} \equiv \frac{\partial \psi}{\partial x}
$$

These equations can be switched for a point sink as well, but the direction of the radial flow will be to the point sink (the opposite direction in Fig. 6).

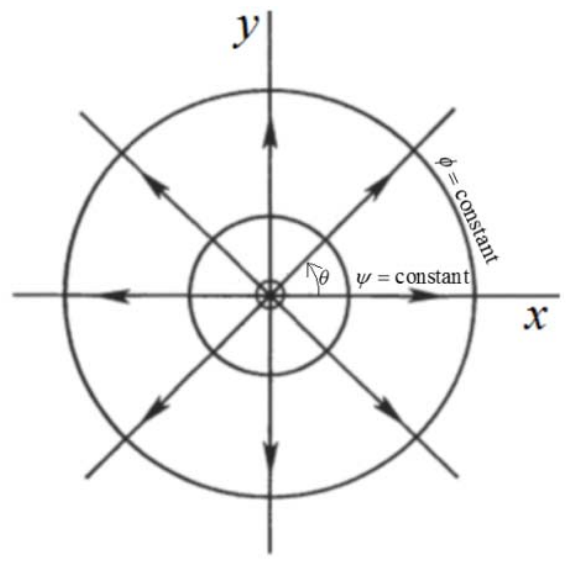

Figure 6. A simple 2D irrotational point source for incompressible flow

\section{Conclusion}

The unanswered questions of our Universe lead the researchers to think about new theories. On the other hand, our nature is a part of our Universe, and the regulations of nature can assist us in developing a new theory. Our nature teaches us about the existence of exact patterns as fractional geometry, Fibonacci patterns, and there are flow patterns in nature, which they approved by constructal law. Also, by observing in nature, we can recognize that everything has an opposite, or anti, or pair, or they are as poles. Therefore, if there was a Big Bang (in this theory a pulse of a point source), it is the possibility of thinking about something else as the opposite side or pair for Big Bang (in this theory the opposite for Big Bang calls sink) from the beginning. In this way, the "Source and Sink theory" indicates, "If there is a source, it must be at least one sink (at the same instance) with a flow direction from the source to the sink, and the process must be conserved". This multi-discipline theory can answer some of the unanswered queries of our Universe. In the special case, if the gravity (curvature of spacetime) of sink sets to zero and consider no space between the source and the sink, this theory will switch to Big Bang theory. This shows the Big Bang theory could be the special case of the application of this multidiscipline new theory for the early Universe. However, source and sink theory has the potential to offer a lot of different scenarios concerning the size and shape of the source $(\mathrm{s}), \operatorname{sink}(\mathrm{s})$ and the space between them to match the available cosmological data better. Consequently, this new theory needs to be considered and completed by the efforts of the researchers of different fields.

\section{References}

1. J. V. Narlikar and N.C. Rana, Hawking process and the cosmic microwave background in a steady-state universe, Physics Letters A, Vol. 72( 2), 1979, pp. 75-77.

2. Anna Ijjas and Paul J. Steinhardt, A new kind of cyclic universe, Physics Letters B, Vol. 795, 2019, pp. 666-672.

3. Robert A. Malaney and Grant J.Mathew, Probing the early universe: a review of primordial nucleosynthesis beyond the standard big bang, Physics Reports, Vol. 229 (4), 1993, pp.145-219.

4. Gasperini M., Veneziano G., The pre-big bang scenario in string cosmology, Physics Reports, Vol. 373, 2003, pp. $1-212$.

5. Ade P. A. R., Aghanim N., Arnaud M., Arroja F., Ashdown M. ..., Planck Collaboration, Astronomy and Astrophysics, A 594, 2016, A20 
6. Nojiri S., Odintsov S.D., Oikonomou V.K., Modified gravity theories on a nutshell: Inflation, bounce and latetime evolution, Physics Reports 692, 2017, pp. 1-104.

7. Kundu P.K., Cohen I.M., Fluid Mechanics, Academic Press, (2002) by Elsevier Science (USA). Anthony Aguirre and Steven Gratton, Inflation without a beginning: A null boundary proposal. Phys. Rev. D, Vol. 67, 2013 2003083515 - Published 29 April 10.1103/PhysRevD.67.083515, Also at: https://arxiv.org/abs/gr-qc/0301042

8. Diana Battefeld and Patrick Peter, A critical review of classical bouncing cosmologies, Physics Reports, Vol. 571, 2015, pp. 1-66.

9. Bahamonde Sebastian, Böhmer Christian G., Carloni Sante, Copeland Edmund J., Fang Wei, Tamanini Nicola, Dynamical systems applied to cosmology: Dark energy and modified gravity, Physics Reports, Vol. 775-777, 2018, pp. 1-122.

10. de Castro L.N., Fundamentals of natural computing: an overview, Physics of Life Reviews, Vol. 4, 2007, pp. 136 .

11. Meakin Paul, Fractal structures, Prog. Solid St. Chem., Vol. 20, 1990, pp. 135--233.

12. Guariglia Emanuel, Harmonic Sierpinski Gasket and Applications, Entropy, Vol. 20, 2018, p. 714.

13. Adrian Bejan and J. Peder Zane, Design in Nature: How the Constructal Law Governs Evolution in Biology, Physics, Technology, and Social Organizations, Anchor publisher, USA, 2012.

14. Alexey Stakhov and Boris Rozin, The "golden" hyperbolic models of Universe, Chaos, Solitons \& Fractals, Vol. 34, 2007, pp. 159-171.

15. Jan C.A. Boeyens, Number patterns in Nature, Crystal Engineering, Vol. 6, 2003, pp. 167-185.

16. Lucia Umberto, Exergy flows as bases of constructal law, Physica A, Vol. 392, 2013, pp. 6284-6287.

17. Cengel Y., Cimbala J.M., Fluid Mechanics: Fundamentals and Applications, McGraw-Hill Education, New York (2016)

18. Cengel, Yunus A., Ghajar, Afshin J., Heat and Mass Transfer: Fundamentals \& Applications, McGraw-Hill USA, fifth edition, 2015.

19. Pereira Thiago, Pitrou Cyril, Isotropization of the universe during inflation, Comptes Rendus Physique, Vol. 16, 2015, pp. 1027-1037.

20. McCoy C.D., Does inflation solve the hot big bang model's fine-tuning problems?, Studies in History and Philosophy of Modern Physics, Vol. 51, 2015, pp. 23-36.

21. Gawiser Eric, Silk Joseph, "The cosmic microwave background radiation", Physics Reports Vol. 333-334, 2000, pp. 245-267.

22. Cirkovi Milan, Perovi Slobodan, "Alternative explanations of the cosmic microwave background: A historical and an epistemological perspective", Studies in History and Philosophy of Modern Physics, Vol. 62, 2018, pp. 118

23. Keskina A.I., Saltib M., Cosmographic nature of the early universe from extra dimensional perspective, Physics Letters B, Vol. 791, 2019, pp. 80-85.

24. Arkani-Hamed Nima, Dimopoulos Savas, Dvali Gia, Large new dimensions and quantum gravity around the corner, Comptes Rendus Physique, Vol. 4, 2003, pp. 347-354.

25. Shapiro Joel A., Taylor Cyrus C., The spacetime supersymmetric formulation of the string, Physics Reports, Vol. 191, Issue 4, 1990, pp. 221-287.

26. Hoyle B., Measuring photometric redshifts using galaxy images and Deep Neural Networks, Astronomy and Computing, Vol. 16, 2016, pp. 34-40.

27. Kundu P.K., Cohen I.M., Fluid Mechanics, Academic Press, (2002) by Elsevier Science (USA). 